\title{
The effects of Roxithromycin on the Activity of APND and GST in Cipangopaludina Chinensis
}

\author{
Hong-wei WANG ${ }^{1,2}$, Duan-bo CAI ${ }^{3}$, Yi-wei LIU $^{1}$, Wen ZHAO ${ }^{1}$, Fang CAII ${ }^{4}$ Ya-peng REN ${ }^{1}, Z e-z h o n g ~ \mathrm{LI}^{1}$ \\ 1. The Key Laboratory of Zoological Systematics and Application, College of Life Science, Hebei University, Baoding, P.R. China \\ 2. South China Institute of Environmental Sciences, MEP, Guangzhou, P.R .China \\ 3. College of Quality and Technical Supervision, Hebei University, Baoding, P.R. China \\ 4. Plant protection station of Beijing City, Beijing, P.R .China
}

\begin{abstract}
Cipangopaludina chinensis which is a kind of benthic invertebrates was taken as experimental invertebrate. In the condition of the Roxithromycin, the activity of APND and GST abstracted from the liver and kidney tissues of Cipangopaludina chinensis to investigate the effect of Roxithromycin on the biochemical indicator of Cipangopaludina chinensis have been tested. The experimental results showed that there were affections on the activity of snail liver and kidney in Cipangopaludina chinensis with Roxithromycin, and the overall trend was promenting at the beginning then suppressing at the end, but the differences of the influence caused by different concentrations of Roxithromycin erythromycin was not obvious. In addition, it appeared from the survival condition of control group and experimental group that Roxithromycin as a kind of antibiotic, has no lethal effect on Cipangopaludina chinensis. But the concentration which is more than 250 times, 500 times and 750 times of the environment used in this experiment had significant influence on the activity of the APND and GST. If the concentration is higher, the influence may be more serious and it is possible to be death.
\end{abstract}

\section{Introduction}

In recent years, thousands of tons of Roxithromycin used for livestock, poultry industry and human health, more and more Roxithromycin get into water throught a series of ways. The growth and development of hydrobios was inhibited. ${ }^{[1]}$ In recent years, thousands of tons of Roxithromycin used for livestock, poultry industry and human health, more and more Roxithromycin get into water throught a series of ways. The growth and development of hydrobios was inhibited. ${ }^{[1]}$. Some tests have found Pearl River cause serious pollution ${ }^{[2]}$, Roxithromycin content of the water is abount in the range of $2 \mathrm{ng} / \mathrm{L}-3000 \mathrm{ng} / \mathrm{L}$, and the sediment is in the range of 2 ng/g-60 ng/g. To better learn the pollution situation of roxithromycin in sediment of the Pearl River, and determine the influence degree on hydrobios Especially the benthos, develop the effective regulation scheme, we conducted the experimental study. The activity of APND and GST abstracted from the stomach and liver tissues of Cipangopaludina chinensis have been tested in the experiment.

\section{Materials and Methods}

\subsection{Experimental materials}

2.1.1 experimental apparatus

Microplate Reader $\quad$ Perlong medical-device firm

\subsubsection{Experimental reagents}

\begin{tabular}{ll}
\hline Roxithromycin(pure) & Toronto Research Chemicals \\
\hline APND ELISA detection kit & $\begin{array}{l}\text { NanjingJiancheng } \\
\text { technology limited }\end{array}$ \\
\hline GST ELISA detection kit & $\begin{array}{l}\text { NanjingJiancheng } \\
\text { technology limited }\end{array}$ \\
\hline
\end{tabular}

\subsection{3 experimental invertebrate parameter}

5 Cipangopaludina chinensis were randomly selected in the tank, and the weight, shell height and shell width were measured. The weight is $3.07 \pm 0.97 \mathrm{~g}$, the shell height is $23.65 \pm 3.45 \mathrm{~mm}$, the shell width is $15.41 \pm 1.71 \mathrm{~mm}$.

There were 5 Cipangopaludina chinensis selected randomly in the tank, and the weight, shell height and shell width were measured. The weight is $3.07 \pm 0.97 \mathrm{~g}$, the shell height is $23.65 \pm 3.45 \mathrm{~mm}$, the shell width is $15.41 \pm 1.71 \mathrm{~mm}$.

\section{2. experimental method}

\subsection{1 raise Cipangopaludina chinensis}

Cipangopaludina chinensis was bought from baiyang lake and raised at water tank which is full of aerated water.The water was changed daily,and the death cipangopaludina chinensis was removed.Then the experiment began two days later.

Cipangopaludina chinensis were devided into 4 group randomized:control group, 250 times Roxithromycin concentration group $(15 \mu \mathrm{g} / \mathrm{g}), 500$ times Roxithromycin concentration group $(30 \mu \mathrm{g} / \mathrm{g}), 750$ times Roxithromycin concentration group $(45 \mu \mathrm{g} / \mathrm{g})$, Each group has 10 individuals $^{[3]}$. Cipangopaludina chinensis raised at white porcelain jars which volume is $2 \mathrm{~L}$. $200 \mathrm{~g}$ sand was put in each jar, mixed Roxithromycin with sand, put $150 \mathrm{ml}$ water ,then added water to the beginning quantity every day. The experiment period is 5 days, gather samples as every day to determine.Cipangopaludina was starved during the experiment in order to excreted most nutrients $[4,5]$.

\subsubsection{Reagent preparation and preliminary experiment}

Dilution or mixing the drug according to the requirements of the kit , and all drugs are stored at $4^{\circ}$ Crefrigerator. 
a) APND ELISA test kit

(1) The standard was diluted by standard diluent into 30 , $60,120,15,7.5 \mathrm{U} / \mathrm{L}$.

(2) $20 *$ Washing buffer dilution: at 1:20 ratio, one $20 *$ washing buffer was added 19 distilled water.

(3)the sample diluent, detection antibody - HRP, substrate $\mathrm{A}, \mathrm{B}$ and terminated liquid were stored at $4{ }^{\circ} \mathrm{C}$ refrigerator Using the Cipangopaludina chinensis with no Roxithromycin to determinate to be familiar with the experimental steps and practice methods.

b) GST ELISA test kit

(1) The standard was diluted by standard diluent into 300 , $150,75,37.5,18.7,0 \mathrm{U} / \mathrm{Lng} / \mathrm{ml}$.

(2) $20 *$ Washing buffer dilution: at 1:20 ratio, one 20* washing buffer was added 19 distilled water.

(3)the sample diluent, detection antibody - HRP, substrate $\mathrm{A}, \mathrm{B}$ and terminated liquid were stored at $4{ }^{\circ} \mathrm{C}$ refrigerator $^{[6]}$.

\subsection{Preparating tissue homogenate}

The Cipangopaludina chinensis was taken randomly from the control group and three experimental groups, the shell was cracked lightly with a hammer and the shell debris were removed with tweezers. Then, organs which is about $0.01 \mathrm{~g}$ were taken from the liver and kidney respectively into the frozen mortar. After dropping 0.99ml double distilled water and grinding, the homogenate would be made. $4 \mathrm{C}$, centrifuged at $3000 \mathrm{rpm}$ for 10 minutes, the supernatant was measured.

\subsection{Determination of biochemical indexes}

\subsubsection{Determination of APND activity}

The APND activity of the organizations in Cipangopaludina chinensis was determined .

(1) The required lath was removed from aluminum foil bag after $20 \mathrm{~min}$ at room temperature,the remaining back to the $4^{\circ} \mathrm{C}$ refrigerator after sealed up

(2)We set up standard, sample and blank hole, standard holes with different concentrations of the standard $50 \mathrm{~L}$, blank hole with nothing.

(3)The sample hole was added $10 \mu \mathrm{L}$ the sample to be tested and $40 \mu \mathrm{L}$ sample dilution.

(4)The standard hole and sample hole were added $100 \mu \mathrm{L}$ antibody marked by HRP.

Then, the reaction hole was sealed by closure plate membrane, 37 constant temperature box for $60 \mathrm{~min}$.

(5)The liquid was abandoned and dried out,then each hole was full of washing liquid, stayed $1 \mathrm{~min}$.Then, the washing liquid was abandoned and dried out,repeated 5 times like this.

(6)All holes were added $50 \mu \mathrm{L}$ substration $\mathrm{A}$ and $\mathrm{B}$,fostered in the darkness in $37^{\circ} \mathrm{C}$ for $15 \mathrm{~min}$.

(7) All holes were added $50 \mu \mathrm{L}$ stop buffer,and the OD value was determined in $450 \mathrm{~nm}$ wavelength by Microplate Reader within $15 \mathrm{~min}$

\subsubsection{Determination of GST activity}

The method of determing the GST activity of the organizations in Cipangopaludina chinensis was like APND.

\subsubsection{The data processing}

Calculating the APND enzyme activity according to the method of kit instructions

Calculating APND and ERND and GST activity in the organizations: In the Excel worksheets, with standard concentration as the abscissa and $450 \mathrm{~nm}$ wavelength corresponding OD value as the ordinate, making the standard linear regression curve. The sample density is calculated according to the curve equation .On the basis of the above formula, different concentration gradient and time gradient of APND and ERND and GST activity were measured, With Excel 2007 and SPSS13.0 to analyze data, the conclusion was drawn.

The experimental results are the average of three times determination on every Cipangopaludina chinensis ,data error line for the standard error, using SPSS statistical software for one way ANOVA (one - way ANOVA), among them, $* \mathrm{P}<0.05$, significant difference was found in the experimental group and control group.

\section{Results}

\subsection{Standard curve}

\subsubsection{APND standard curve}

TABLE I THE DETERMINATION TABLE OF APND STANDARD CURVE

\begin{tabular}{ccccccc}
\hline $\begin{array}{c}\text { Concentrati } \\
\text { on (U/L) }\end{array}$ & 0 & 7.5 & 15 & 30 & 60 & 120 \\
\hline OD value & 0.047 & 0.121 & 0.234 & 0.426 & 0.874 & 1.504 \\
\hline
\end{tabular}

According to the data in the table,we got APND standard curve using Excel:

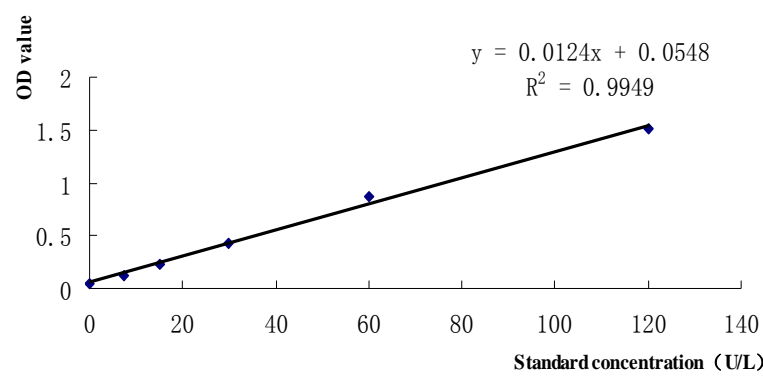

Fig. 1 APND standard curve 


\subsubsection{GST standard curve}

TABLE II THE DETERMINATION TABLE OF GST STANDARD CURVE

\begin{tabular}{ccccccc}
\hline $\begin{array}{c}\text { Concentration } \\
(\mathrm{U} / \mathrm{L})\end{array}$ & 0 & 18.7 & 37.5 & 75 & 150 & 300 \\
\hline OD value & 0.037 & 0.067 & 0.17 & 0.30 & 0.74 & 1.26 \\
\hline
\end{tabular}

According to the data in the table, we got GST standard curve using Excel:

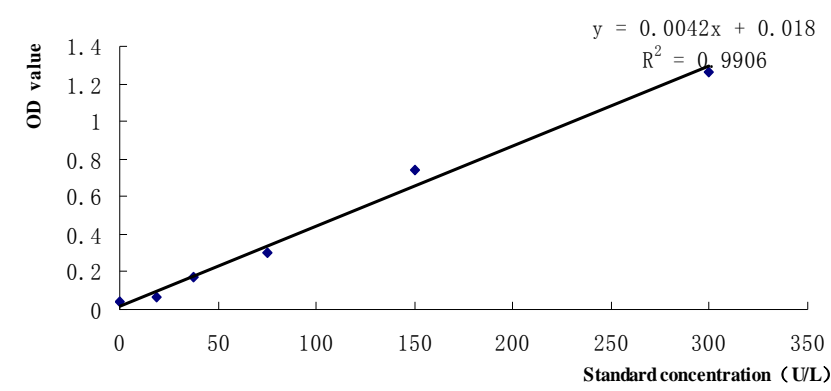

Fig. 2 GST standard curve

\subsection{The Activities of Enzyme in Liver}

3.2.1 The changes of ERND activity in liver of $C$. chinensis

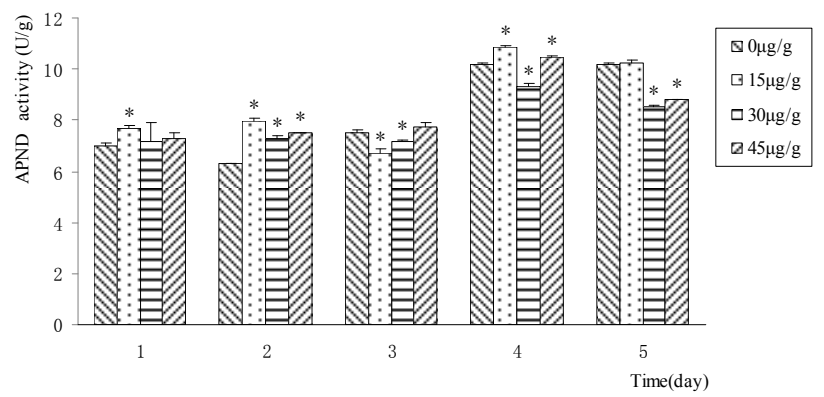

Fig. 3 The changes of APND activity in liver of C. chinensis (* $P<0.05$, extremely significantly differences between treatments and the control group)

3.2.2 The changes of GST activity in liver of C. chinensis

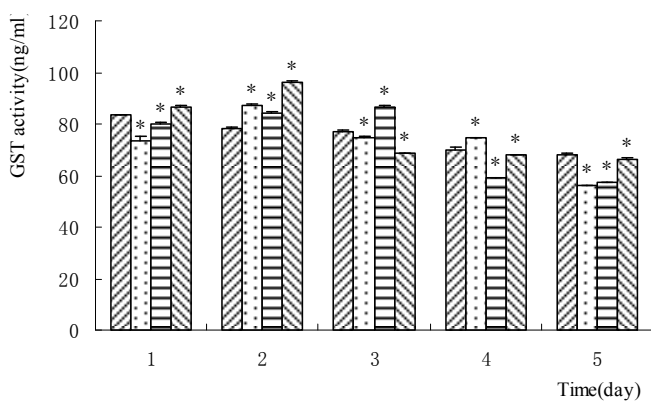

$\square 0 \mu \mathrm{g} / \mathrm{g}$

$\square 30 \mu \mathrm{g} / \mathrm{g}$

⿶ $45 \mu \mathrm{g} / \mathrm{g}$

Fig. 4 The changes of GST activity in liver of $C$. chinensis $(* P<0.05$, extremely significantly differences between treatments and the control group)

\section{3 The Activities of Enzyme in Liver kidney}

\subsubsection{The changes of APND activity in kidney}

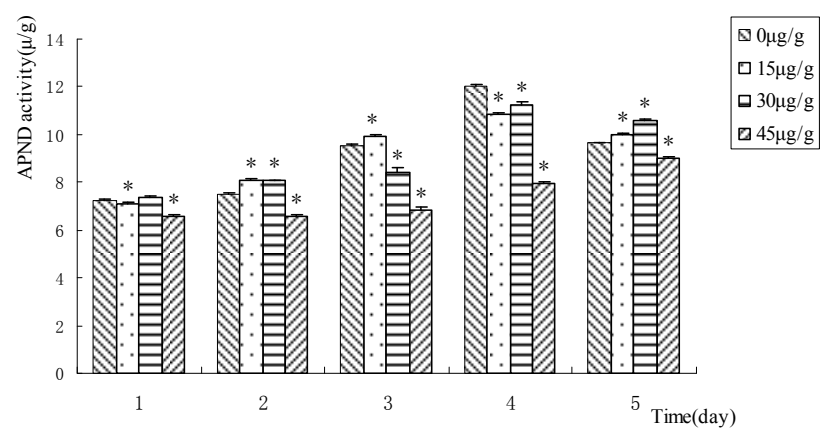

Fig. 5 The changes of APND activity in kidney of C. chinensis (* $P<0.05$, extremely significantly differences between treatments and the control group)

\subsubsection{The changes of GST activity in kidney}

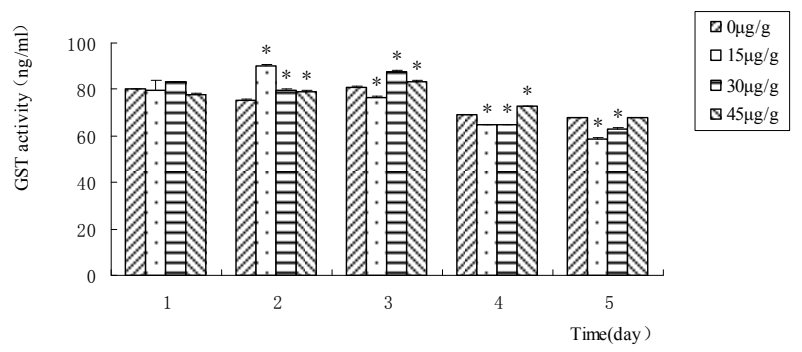

Fig. 6 The changes of GST activity in kidney of C. chinensis (* $P<0.05$, extremely significantly differences between treatments and the control group)

\section{Discussion}

The experimental has studied the effect of roxithromycin on the activity of APND and GST in Cipangopaludina chinensis's liver and kidney. The results show that the effect trend of roxithromycin on the activity of APND and GST in different tissues is roughly same. In general, the overall trend was promenting at the beginning then suppressing at the end. But in the kidney, APND activity of $45 \mu \mathrm{g} / \mathrm{g}$ roxithromycin group showed an ascending trend. It not decreased in the last day. May be the high concentration of roxithromycin has serious effect on kidney of cipangopaludina chinensis. In muscle, APND inhibitory effect is not very obvious. $45 \mu \mathrm{g} / \mathrm{g}$ roxithromycin group also show no inhibition effect. May be due to muscle taken from cipangopaludina chinensis foot. Foot had close contacts with Sand mixed with roxithromycin. It has serious toxicity on foot, APND activity show no decrease or the trend of decreasing trend is not obvious. Different concentrations of roxithromycin on the various organizations of cipangopaludina chinensis in APND and GST activity is not great. May be because the small roxithromycin concentration gradient is not sufficient to cause great changes. But the environmental concentration of roxithromycin in sediments is not very 
high. Setting too high concentration of roxithromycin does not have great practical significance to the experiment research purposes.

\section{Conclusion}

In the condition of the Roxithromycin, there were varying degrees affectionsthe on the activity of APND and GST from the liver and kidney tissues of Cipangopaludina chinensis, and the overall trend was promenting at the beginning then suppressing at the end, but the differences of the influence caused by different concentrations of Roxithromycin erythromycin was not obvious. In addition, it appeared from the survival condition of control group and experimental group that Roxithromycin as a kind of antibiotic, has no lethal effect on Cipangopaludina chinensis. But the concentration which is more than 250 times, 500 times and 750 times of the environment used in this experiment had significant influence on the activity of the APND and GST. If the concentration is higher, the influence may be more serious, and it is possible to be death.

Therefore, we must pay enough attention to the pollution caused by the abuse of antibiotics such as Roxithromycin, manage and govern timely, to avoid harm out of control. In short, governance against the use of antibiotics and management of pollution elements has an extremely important practical significance. The management methods on Roxithromycin pollution needs further study.

\section{Acknowledgment}

This research was supported by NSFC U1133005; Subject of the strong characteristic Biology in Hebei Province; This research was supported by NSFC U1133005; The natural science foundation of Hebei Province(D2013201105); The Education Department of Hebei province key project(2013-77); State Education
Ministry (SRF for ROCS, SEM2014); Hebei Characteristics of Seafood Innovation Team of Modern Agro-industry Technology Research System; Projects of coastal habitat restoration in Qinhuangdao (Provincial Science and Technology Department, No. 15273303D); Hebei Provincial Key Laboratory of operating subsidy (item number: 14967611D). Funded by Heibei Province construction project in Strong and characteristic disciplines of biology; Funded by Hebei Province project for cultivating national key disciplines in biology; Funded by Hebei University construction project for comprehensive strength promotion of Midwest colleges and universities.

\section{References}

[1] Yi Zou,Haibo Li. Research and Progress of Antibiotic Elimination in the Environment[A]. University of Shanghai for Science and Technology (USST), 2011:5.

[2] CHANG ChihChung,CHOU C.K.Charles,Andreas Wahner. Regional ozone pollution and key controlling factors of photochemical ozone production in Pearl River Delta during summer time [J]. Science China (Chemistry), 2010, 03:650-662.

[3] Qian Chen, Dinglong Li."'Remediation of Eutrophic Water Body by Zoobenthos", 2010 International Conference on Future Biomedical Information Engineering

[4] Biological coupling anti-wear properties of three typical molluscan shells-Scapharca subcrenata, Rapana venosa and Acanthochiton rubrolineatus[J]. Science China (Technological Sciences), 2010, 11:2905-2913.

[5] Effect of periphyton community structure on heavy metal accumulation in mystery snail (Cipangopaludina chinensis):A case study of the Bai River,China[J]. Journal of Environmental Sciences, 2012, 10:1723-1730. 